\title{
Case study: Use of contact lenses to manage complications following combined corneal cross-linking and topography-guided photorefractive keratectomy for pellucid marginal degeneration
}

\author{
D. Yeung, OD, BSc, \\ MSc candidate \\ School of Optometry \\ and Vision Science, \\ University of Waterloo

\section{Luigina Sorbara} \\ School of Optometry \\ and Vision Science, \\ University of Waterloo
}

\begin{abstract}
This case report describes two significant long-term complications experienced by a patient following treatment for pellucid marginal degeneration (PMD). Two years after undergoing a combination of topography-guided photorefractive keratectomy (T-PRK) and corneal collagen cross-linking (CXL) procedures, the patient continued to experience glare and dryness associated with persistent stromal haze and dry eye. These procedures resulted in dissatisfaction with the final outcome, which led the patient to seek contact lens correction. Management of the symptomatic ocular sequelae with specialty soft toric contact lenses designed for irregular corneas supported her visual rehabilitation.

KEY WORDS:

topography-guided photorefractive keratectomy (T-PRK), corneal collagen cross-linking (CXL), Pellucid Marginal Degeneration (PMD), stromal haze, dry eye
\end{abstract}


$\mathrm{P}$ ellucid marginal degeneration (PMD) is a progressive non-inflammatory ectatic disorder involving the inferior peripheral region of the cornea. ${ }^{1-3}$ In this disorder, thinning occurs in a crescent shape extending from the 4 o'clock to 8 o'clock position in the peripheral cornea. While the amount of irregular astigmatism depends on the severity of the condition, the vision distortion secondary to PMD is typically asymptomatic in the early stages because the area of thinning does not involve the visual axis. For this reason, corneal topography is the gold standard for diagnosis of this condition. 3 The current standard of practice in the management of PMD includes spectacles, contact lenses and surgical interventions, such as lamellar or penetrating keratoplasty.1, ${ }^{4,5}$ Recently, simultaneous topography-guided photorefractive keratectomy (T-PRK) and corneal collagen cross-linking (CXL) procedures have been used to manage PMD and other causes of keratoectasia with the aim of slowing the progression, minimizing corneal irregularity, and reducing the need for a corneal transplant. ${ }^{6-10}$

T-PRK involves ablation of the corneal stromal tissue by an excimer laser with customization based on wavefront aberrometry, tomography, and topography. ${ }^{11-13}$ By flattening the corneal apex or elevated and steepened tissue adjacent to ectatic areas, this procedure normalizes the cornea by reducing the astigmatism and irregularity of the corneal surface. ${ }^{6,11,13} \mathrm{CXL}$ uses the photosensitization of riboflavin with ultraviolet A (UVA) light to induce the polymerization of collagen fibers in the anterior 200 to 300 microns of the corneal stroma. ${ }^{14-18} \mathrm{CXL}$ effectively increases the corneal rigidity and the biomechanical strength by three-fold.,17,19 $\mathrm{CXL}$ also results in simultaneous flattening of the steepest area and steepening of adjacent areas around the cone, which decreases corneal asymmetry and spherical aberration. ${ }^{19}$ The combination of these procedures, with CXL performed immediately after T-PRK, is beneficial in the management of corneal ectasia. ${ }^{8-10}$ It has been demonstrated that the operating time can be reduced if these procedures are performed simultaneously. ${ }^{20}$ This also allows for riboflavin to reach and induce collagen cross-linking in the deeper layers of stromal tissue. ${ }^{20}$ It minimizes the potential for superficial stromal scarring resulting from PRK by diminishing fibroblast activity after the eradication of keratocytes. ${ }^{6}$ Reversing the order of these two procedures would result in either the removal of stiffened cross-linked corneal tissue or a reduction in the ablation rate of cross-linked tissue with altered biomechanical properties, neither of which would be beneficial. ${ }^{8,10,20}$

The effectiveness of simultaneous T-PRK and CXL in halting the progression of ectasia over a limited period of time and improving uncorrected and best-corrected visual acuities for PMD has been well documented. ${ }^{8,10}$ However, little information is available regarding the complications from these procedures. This case report describes two such complications (permanent corneal stromal haze and dry eye) and their management.

\section{CASE REPORT}

A 41-year-old Caucasian female presented at the University of Waterloo School of Optometry and Vision Science Contact Lens Clinic for a consultation regarding specialty contact lens options. Her chief visual concerns included significant light sensitivity and glare, both of which were severe enough to have impacted her daily activities. Her ocular history was significant for bilateral PMD that had been diagnosed five years previously at a refractive surgery consultation appointment in 2010. Shortly after her diagnosis, she underwent simultaneous T-PRK and CXL in both eyes. The patient attributed her debilitating visual symptoms and dry eye to these procedures. Overall, her general heath was unremarkable other than the occasional migraine. A previous medical examination had ruled out Sjogren's syndrome. Her current medications included cyclosporine (Restasis'), duloxetine (Cymbalta), lorazepam, and zopiclone (Imovane').

Other significant ocular and contact lens history included prior experience with gas permeable and disposable soft lenses, with little success primarily due to her severe symptoms of dryness, which eventually led to contact lens intolerance. She had previously been prescribed a series of standard dry eye managements, including lid hygiene and hot compresses, frequent application of artificial tears with and without lipid components, a one-month course of topical loteprednol, a two-year course of topical cyclosporine A and bilateral inferior punctal occlusion. None of these prior therapies had restored the patient's visual comfort to a level that allowed her to perform daily functions.

Upon ocular examination, refraction was $-0.75 /-2.50 \times 148\left(6 / 9^{-2}\right)$ in her right eye and $-0.25 /-2.25 \times 057\left(6 / 9^{+2}\right)$ in her left eye. All entrance tests were unremarkable. Her pupils were equal in size at approximately $8.0 \mathrm{~mm}$ under scotopic conditions and $5.0 \mathrm{~mm}$ under photopic conditions.

Biomicroscopic examination of the anterior segment revealed central stromal haze in both eyes with significant confluent staining of the epithelium of the inferior cornea, which was graded at 2+. Bilateral central stro- 
mal haze was also observed using an anterior segment optical coherence tomography (OCT) imaging system (Visante $^{\mathrm{Tm}}$, Zeiss, Jena, Germany) (Figure 1A shows the right eye and Figure 1B shows the left eye). Furthermore, the demarcation line as a result of the CXL procedure could be observed and the corneal haze extended through the anterior and posterior stroma. Mild to moderate meibomian gland dysfunction was noted in both eyes. Tear breakup times (TBUT) were 4 seconds and 5 seconds in the right and left eyes, respectively. A phenol red thread test (PRTT) gave results of $22 \mathrm{~mm}$ in the right eye and $18 \mathrm{~mm}$ in the left eye, which ruled out aqueous-deficient dry eye.

Figure $1 A$

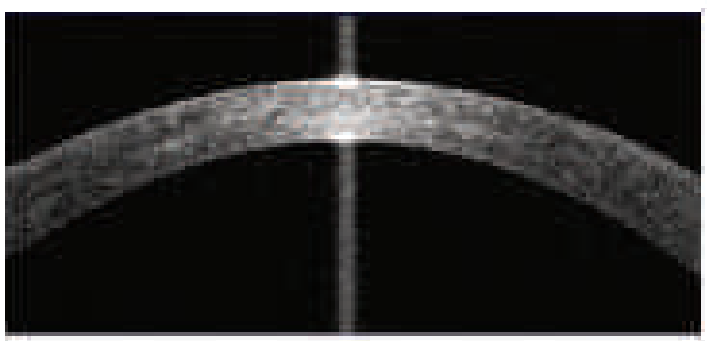

Figure $1 B$

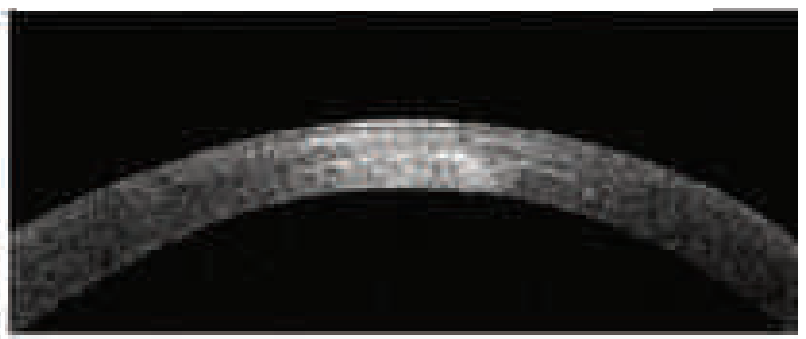

Corneal topographies obtained with a Pentacam ${ }^{\mathrm{TN}}$ system (Oculus, Wetzlar, Germany) revealed simulated keratometry values of 38.4D@168\% / 40.0D in the right eye and 38.8D@67.5\% 39.6D in the left eye (Figure 2).

Figure $2 A$

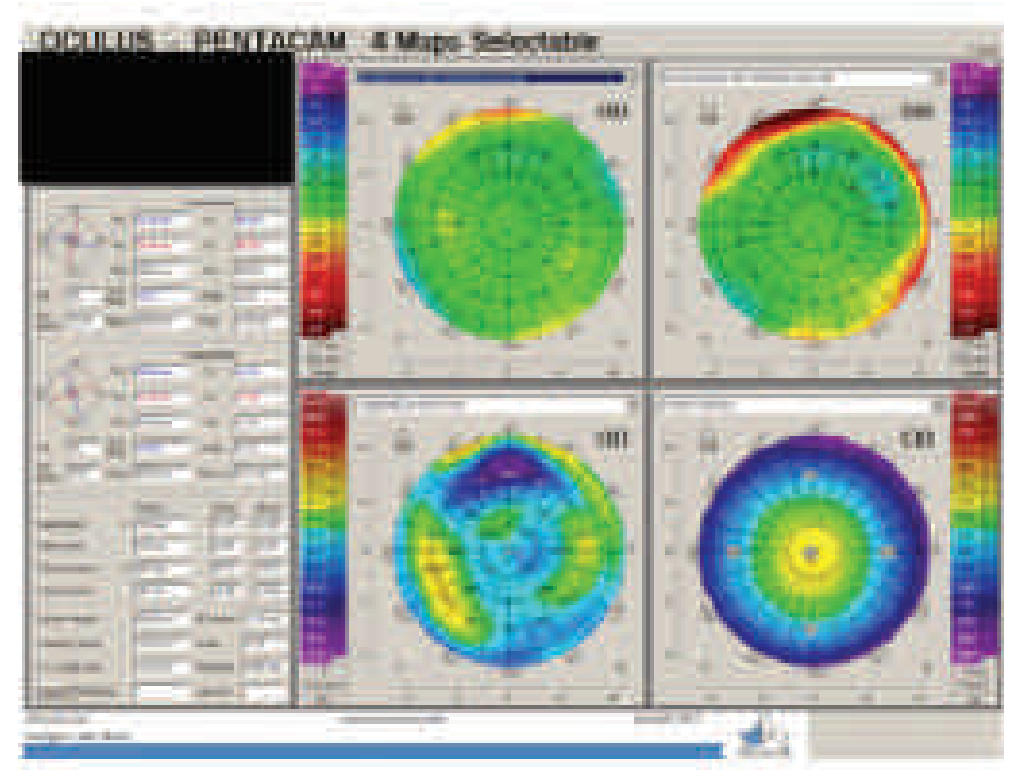


Figure $2 B$

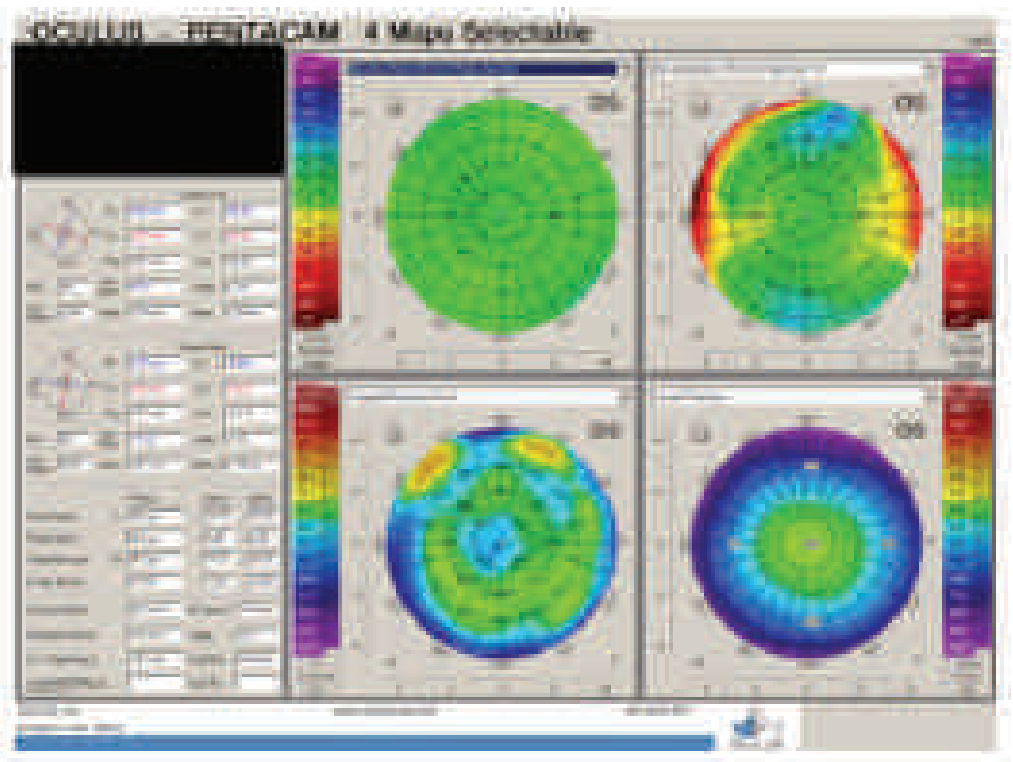

According to her prior medical records at the refractive surgery consultation, her baseline simulated keratometry readings were 43.00D@097\% 45.00 in the right eye and 45.00D@015\% 46.00 in the left eye. Pachymetry results revealed a thinnest-point reading of $564 \mu \mathrm{m}$ in the right eye and a thinnest-point reading ranging from $247 \mu \mathrm{m}$ to $555 \mu \mathrm{m}$ with large variability across multiple scans in the left eye. Mild-to-moderate meibomian gland dysfunction without corneal abnormalities was noted on an anterior segment health check.

We concluded that the patient exhibited visual symptoms secondary to corneal haze as a result of having undergone T-PRK and CXL, as well as evaporative dry eye symptoms that worsened following the procedure. After extensive discussions with the patient on management options, we decided that she might benefit from specialty contact lenses. The initial specialty contact lens option considered for this patient was scleral lenses. In anticipation of easier adaptation, we chose a scleral lens with a smaller overall diameter and a thinner center thickness. According to the Rose K2 XL (Blanchard Laboratories, Sherbrooke, Quebec) fitting guide21, we selected the following diagnostic lenses:

OD: Rose K2 XL / 8.00/ 14.6/ plano/ Standard periphery

OS: Rose K2 XL / 8.10/ 14.6/ plano/ Standard periphery

The lenses were applied and a settling time of 30min was allowed. The lens fitting characteristics were then examined. Both lenses demonstrated excessive clearance centrally and minimal clearance limbally. The lenses were well-centered and provided good coverage. Mild edge lift was noted in both eyes at 3 and 9 o'clock. The patient also reported mild-moderate lens awareness in both eyes. The fit of the lenses was deemed to be too steep centrally. Based on the assessment and over-refraction of the trial lenses, we placed a final order with the following parameters:

OD: Rose K2 XL / 8.00/ 14.8/ -4.00/ Decreased edge lift \#1

OS: Rose K2 XL / 8.30/ 14.8/ -2.75/ Decreased edge lift \#1 


\section{Follow-up \#1}

At the lens delivery appointment, the fit of the lenses was deemed appropriate, with adequate central clearance. The patient continued to report mild-moderate lens awareness. She was counselled that initial lens awareness is appropriate for a novice scleral lens wearer, and informed to slowly increase wear time to ease the adaptation process, beginning with two to three hours daily until her next follow-up appointment. She was also instructed how to properly insert and remove the scleral lenses along with proper handling techniques. The lenses were dispensed to the patient along with a starter kit of Daily Boston Simplus * solution (Bausch and Lomb, Rochester, NY).

\section{Follow-up \#2}

At the two-week follow-up appointment, lens assessment using a biomicroscope confirmed that the lenses were well centered with an appropriate apical clearance of approximately 75 microns in accordance with the Rose K2 XL fitting manual.21 Mild, but tolerable, compression in the limbal zone was noted superiorly and inferiorly in both eyes. The spherocylindrical over-refraction was $+1.00-2.00 \times 105(6 / 7.5)$ in the right eye and $+0.25-1.00 \times 090\left(6 / 7.5^{-3}\right)$ in the left eye. However, while the patient reported a reduction in her symptoms of dryness with lens wear, she was dissatisfied with the general comfort of the contact lenses, and noted greater lens awareness in the left eye compared to the right eye. Ultimately, we concluded that the patient should discontinue the use of scleral lenses due to inadequate comfort and poor adaptation to this type of lens.

The patient was refitted with Kerasoft ${ }^{\circ}$ IC (Bausch + Lomb), a custom soft keratoconic lens design, as an alternative for the management of her visual symptoms. We ordered lenses with the following parameters:

OD: Kerasoft ${ }^{\circ}$ IC / 8.20/ 14.5/ -0.50 -0.75 x 052 / Flat \#1 periphery

OS: Kerasoft ${ }^{\circledR}$ IC / 8.60/ 14.5/ -1.25 -1.00 x 075/ Standard periphery

\section{Follow-up \#3}

At the two-week follow-up appointment for the Kerasoft ${ }^{\circ}$ IC lenses, the patient was quite content and reported a subjective improvement in the quality of her vision with lens wear and no eye fatigue. She reported good overall comfort with the lenses. However, she was only able to wear her lenses for two hours per day, after which her vision became foggy. She was using Biotrue ${ }^{\mathrm{TM}}$ multipurpose solution (Bausch + Lomb) to clean and store the lenses on a nightly basis. The patient achieved visual acuities of $6 / 7.5$ in the right eye and 6/9+ in the left eye with no significant improvement in vision with a spherical over-refraction. In a lens assessment, both lenses were observed to be well centered with adequate movement on primary gaze and upgaze. The rotation was stable at 5 degrees counter-clockwise in the right eye and 12 degrees counter-clockwise in the left eye. A moderate amount of lipid deposits was found on her lenses. At the end of the appointment, we decided that she should continue with her lenses with no changes in the parameters, but to switch her care system to Peroxi$\operatorname{clear}^{\mathrm{TM}}$ (Bausch + Lomb). Complete Blink and Clean ${ }^{\mathrm{TM}}$ eye drops (Abbott Medical Optics, Abbott Park, IL) were recommended as needed during contact lens wear.

\section{Follow-up \#4}

The patient reported good results with Kerasoft IC lenses. Her wear time had increased to seven hours per day for three to four days per week with the change in her care system and the use of Complete Blink and Clean ${ }^{\mathrm{Tm}}$ eye drops (Abbott Medical Optics) four times daily. The patient continued to experience subjective glare with the lenses, likely as a result of her large pupil size in photopic and scotopic conditions and the significant corneal haze secondary to the simultaneous T-PRK and CXL procedures. The custom soft keratoconic lenses successfully restored her functional vision for normal daily activities. She will be followed-up quarterly.

\section{DISCUSSION}

Pellucid marginal degeneration (PMD) is a progressive non-inflammatory keratoectasia resulting in inferior corneal thinning and irregular astigmatism. ${ }^{1-3}$ The use of gas permeable corneal and scleral contact lenses can correct the irregular astigmatism that is induced as the condition progresses and the cornea thins. It has been shown that simultaneous T-PRK and CXL can increase the effectiveness of CXL by allowing better penetration of the riboflavin solution used with CXL, due to the absence of Bowman's layer and a partially ablated stroma. ${ }^{20}$ The use of simultaneous procedures may also reduce the sub-epithelial haze that commonly follows PRK. ${ }^{6}$ The combined T-PRK and CXL technique can be an effective option for the management of PMD and can improve the prognosis by reducing the amount and irregularity of the corneal astigmatism. However, little 
information is available regarding complications and patient satisfaction following the procedure. This case study presented a 41-year-old female patient who exhibited clinically significant permanent corneal haze and dry eye symptoms after undergoing combined T-PRK and CXL.

Permanent corneal haze following CXL is defined as a stromal opacity that persists for more than six months postCXL, and has been documented in $5 \%$ to $8.6 \%$ of cases. ${ }^{22,23}$ The corneal opacity that follows CXL typically spans the anterior 300 microns of stroma and can be differentiated from the sequelae post-PRK because the latter is sub-epithelial and much shallower. ${ }^{15,18,24,25}$ Furthermore, this long-term CXL complication is differentiated from the transient lacunar honeycomb-like central opacity that occurs within the anterior 300 microns of the corneal stroma present in all eyes at 1-month post-CXL. ${ }^{26,27}$ The presence of a transient haze, which only extends throughout the depth of the corneal stroma affected by CXL, is an expected indication of a successful CXL procedure. With the use of topical preservative-free steroid therapy, which is typically prescribed for 1 to 3 months postoperatively, the decrease in corneal transparency subsides over 6 months to 1 year following CXL, and coincides with the completion of keratocyte repopulation..$^{22,27}$ On the other hand, a permanent stromal haze reflects changes in crystalline proteins and lamellar interconnections induced by the interaction of riboflavin and ultraviolet A radiation. ${ }^{28,29}$ While this steroid-resistant haze has not been shown to cause any reduction in high-contrast visual acuity, it may increase the backscatter of light that is internally reflected within the cornea. ${ }^{22,29-31}$ Thus, it may have led to the degradation of visual quality and the debilitating symptoms of glare and visual distortion due to these aberrations experienced by the patient in this case. Raiskup et al. noted a higher risk of permanent stromal haze following CXL in cases of severe keratoectasia with steeper pre-operative keratometric values. ${ }^{23}$ These cases likely presented with intrinsic stromal anomalies associated with keratoectasia, such as micro-striae and hyper-activated keratocyte nuclei in the stromal tissue, and with the presence of Vogt's striae prior to the CXL procedure. ${ }^{23}$

Regarding possible treatments, no studies to date have investigated the potential benefits of reducing the post-CXL stromal haze with the use of mitomycin $\mathrm{C}$, an anti-proliferative agent that has been shown to reduce the risk of post-PRK corneal haze. ${ }^{32}$ However, the use of mitomycin $\mathrm{C}$ in patients with keratoectasia may be potentially contraindicated due to the cytotoxic properties of mitomycin $\mathrm{C}$, and the role it plays in inducing keratocyte apoptosis may increase the risk of accelerating further corneal thinning.

Our patient exhibited significant corneal haze that spanned the full stromal thickness in both eyes, as demonstrated in the anterior segment OCT scans in Figure 1. The scans also demonstrate a demarcation line through the stroma that signifies the extent of anterior stroma that had been affected by CXL. 15,18,25-27,30,33 This presentation of stromal opacity that extends into the posterior stroma is atypical of post-CXL stromal haze. Güell et al. reported posterior stromal haze developing 5 months after simultaneous T-PRK and CXL in a 22-year-old patient with forme fruste keratoconus. ${ }^{34}$ In another case series, linear hyper-reflective structures were noted in 13 of 28 eyes that had undergone simultaneous TPRK and CXL for the management of keratoconus.35 However, the authors did not find any correlation between the prevalence of posterior stromal haze and the patient's preoperative corneal thickness or ablation depth.

Dryness after kerato-refractive surgery is one of the primary causes of patient post-operative dissatisfaction..$^{36}$ Interruption of the neural feedback system due to a reduction in corneal sensitivity has been proposed to be the primary cause of dry eye after kerato-refractive surgery. ${ }^{37,38}$ In the case of combined T-PRK and CXL, the epithelium is removed using either topical alcohol, mechanical scraping, a rotating brush, or a laser, followed by irradiation of the anterior 300 microns of stromal tissue with UVA. ${ }^{20,39,40}$ This procedure leads to disruption of the sub-epithelial and anterior-mid stromal nerve plexus, resulting in transiently reduced lacrimal gland production within the first six months post-operatively. ${ }^{29,38,39}$ Regeneration of the sub-epithelial plexus from the surrounding non-irritated area begins at the end of the first month, while extension of the deeper stromal nerve plexus into the anterior stroma can be observed as early as the second to third month. ${ }^{29}$ With the use of confocal microscopy, studies have confirmed that the number of nerve fibres is essentially restored to preoperative baseline values by six months after the procedure, but the interconnections between neural fibres continue to increase up until two years after the procedure. ${ }^{29,41}$ Corneal sensitivity returns to its preoperative baseline within one year after the procedure, and basic tear secretion and tear film stability should be restored concurrently. ${ }^{29,42,43}$

In this patient, PRTT of greater than $15 \mathrm{~mm}$ was measured in both eyes along with a decreased TBUT, which suggested the presence of other factors that may have contributed to the signs and symptoms of dryness. Firstly, the cornea takes on an oblate topography after undergoing kerato-refractive surgery. The irregularity of the corneal surface, as confirmed by the presence of an oblique corneal cylinder, may act as an obstacle for the even distribution of the tear 
film with blinking. Secondly, the discrepancy between reported symptoms and signs of dryness can be explained by an altered excitability in regenerated nerves caused by the induced inflammatory process after corneal nerve injury. ${ }^{44}$ Thirdly, the long-term use of antidepressant and antianxiety medications has been known to have an anti-cholinergic effect, which results in decreased aqueous production and dry eye symptoms. ${ }^{45,46}$ Finally, a natural decrease in goblet cell population with age can contribute to a compromised mucin layer that results in the instability of the tear film..$^{39,47}$

In this case study, a 41-year-old patient underwent simultaneous T-PRK and CXL shortly after a diagnosis of PMD. For a patient who was visually asymptomatic prior to her diagnosis, her quality of life is arguably decreased postoperatively due to her debilitating symptoms of glare, light sensitivity, and dryness. Bilateral and permanent postCXL corneal haze and the resultant increase in internal reflectivity (light scatter) within the stromal tissue are responsible for her visual symptoms. These symptoms are likely exacerbated by her larger-than-average scotopic and photopic pupil sizes of $8 \mathrm{~mm}$ and $5 \mathrm{~mm}$, respectively. The T-PRK and CXL procedures exacerbated her dry eye symptoms; she was already at high risk given her age, a pre-operative history of meibomian gland dysfunction and the use of an oral contraceptive, and her current use of antidepressant and anti-anxiety medications. While this patient is now being successfully managed with specialty soft toric lenses for her irregular cornea, careful screening pre-operatively, while paying special attention to pupil size and ocular history, may help identify patients who are at higher risk of developing undesirable outcomes.

\section{CONCLUSION}

Simultaneous T-PRK and CXL can effectively improve corrected and uncorrected visual acuities while slowing the progression of PMD and other keratoectasiae. ${ }^{8,10}$ This combination may effectively delay the need for more invasive management such as penetrating keratoplasty and implantation of intrastromal ring segments. ${ }^{17}$ This case report reviewed two significant long-term complications after these procedures (glare associated with persistent stromal haze and chronic dryness), which resulted in the patient's dissatisfaction with the outcome. A more careful consideration of inclusion criteria for these procedures may ultimately increase the relative benefits of slowing the progression of PMD while decreasing the relative risks of complications. 


\section{REFERENCES:}

1. Sridhar MS, Mahesh S, Bansal AK, et al. Pellucid marginal corneal degeneration. Ophthalmology 2004;111:1102-7. doi:10.1016/j. ophtha.2003.09.035.

2. Krachmer JH. Pellucid marginal corneal degeneration. Arch Ophthalmol 1978;96(7):1217-21.

3. Jinabhai A, Radhakrishnan H, O'Donnell C. Pellucid corneal marginal degeneration: A review. Contact Lens Anterior Eye 2011;34(2):56-63. doi:10.1016/j.clae.2010.11.007.

4. Biswas S, Brahma A, Tromans C, et al. Management of pellucid marginal corneal degeneration. Eye 2000;14(4):629-34. doi:10.1097/01.ico.0000153555.82278.5b.

5. Varley GA, Macsai MS, Krachmer JH. The results of penetrating keratoplasty for pellucid marginal corneal degeneration. Am J Ophthalmol 1990;110(2):149-52.

6. Kanellopoulos AJ. Comparison of sequential vs same-day simultaneous collagen cross-linking and topography-guided PRK for treatment of keratoconus. J Refract Surg 2009;25(9):S812-8. doi:1 0.3928/1081597X-20090813-10.

7. Wollensak G, Spoerl E, Seiler T. Stress-strain measurements of human and porcine corneas after riboflavin-ultraviolet-Ainduced cross-linking. J Cataract Refract Surg 2003;29:1780-5 doi:10.1016/S0886-3350(03)00407-3.

8. Kymionis GD, Karavitaki AE, Kounis GA, et al. Management of pellucid marginal corneal degeneration with simultaneous customized photorefractive keratectomy and collagen crosslinking. J Cataract Refract Surg 2009;35(7):1298-301. doi:10.1016/j. jcrs.2009.03.025.

9. Stojanovic A, Zhang J, Chen X, et al. Topography-guided transepithelial surface ablation followed by corneal collagen cross-linking performed in a single combined procedure for the treatment of keratoconus and pellucid marginal degeneration. J Refract Surg 2010;26(2):145-52. doi:10.3928/108159 7X-20100121-10.

10. Kymionis GD, Kontadakis GA, Kounis GA, et al. Simultaneous topography-guided PRK followed by corneal collagen cross-linking for keratoconus. J Refract Surg 2009;25(September):807-12. doi:10.3928/1081597X-20090813-09.

11. Kanellopoulos AJ. Managing highly distorted corneas with topography-guided treatment. ISRS/AAO 2007 Subspecialty Day/Refractive Surg Syllabus Sect II Ablation Strategies 2007:135 .

12. Cennamo G, Intravaja A, Boccuzzi D, et al. Treatment of keratoconus by topography-guided customized photorefractive keratectomy: Two-year follow-up study. J Refract Surg 2008;24(2):145-9.

13. Lin DTC, Holland SR, Rocha KM, et al. Method for optimizing topography-guided ablation of highly aberrated eyes with the ALLEGRETTO WAVE excimer laser. J Refract Surg 2008;24(4):S439-45.

14. Caporossi A, Mazzotta C, Baiocchi S, et al. Long-term results of riboflavin ultraviolet A corneal collagen cross-linking for keratoconus in Italy: The Siena Eye Cross Study. Am J Ophthalmol 2010;149(4):585-93. doi:10.1016/j.ajo.2009.10.021.

15. Dhawan S, Rao K, Natrajan S. Complications of corneal collagen cross-linking. J Ophthalmol 2011;2011:869015. doi:10.1155/2011/869015

16. Spadea L. Corneal collagen cross-linking with riboflavin and UVA irradiation in pellucid marginal degeneration. J Refract Surg 2010;26(5):375-7. doi:10.3928/1081597X-20100114-03.

17. Wollensak G, Spoerl E, Seiler T. Riboflavin/ultraviolet-A-induced collagen crosslinking for the treatment of keratoconus. Am J Ophthalmol 2003;135:620-7. doi:10.1016/S0002-9394(02)02220-1.

18. Dahl BJ, Spotts E, Truong JQ. Corneal collagen cross-linking: An introduction and literature review. Optometry 2012;83(1):33-42. doi:10.1016/j.optm.2011.09.011.

19. Vinciguerra P, Albè E, Trazza S, et al. Refractive, topographic, tomographic, and aberrometric analysis of keratoconic eyes undergoing corneal cross-linking. Ophthalmology 2009;116(3):369 78. doi:10.1016/j.ophtha.2008.09.048.

20. Kanellopoulos AJ, Binder PS. Management of corneal ectasia after LASIK with combined, same-day, topography-guided partial transepithelial PRK and collagen cross-linking: the athens protocol. J Refract Surg 2011;27(5):323-31. doi:10.3928/108159 7X-20101105-01.
21. Blanchard Contact Lens Inc. Rose K2 XL Semi-scleral lens Fitting Guide. Canada (Toronto, ON).

22. Mazzotta C, Balestrazzi A, Traversi C, et al. Treatment of progressive keratoconus by riboflavin-UVA-induced cross-linking of corneal collagen: ultrastructural analysis by Heidelberg Retinal Tomograph II in vivo confocal microscopy in humans. Cornea 2007;26:390-7. doi:10.1097/ICO.0b013e318030df5a.

23. Raiskup F, Hoyer A, Spoerl E. Permanent corneal haze after riboflavin-UVA-induced cross-linking in keratoconus. J Refract Surg 2009;25(9):S824-8. doi:10.3928/1081597X-20090813-12.

24. Eliacik M, Bayramlar H, Erdur SK, et al. Anterior segment optical coherence tomography evaluation of corneal epithelium healing time after 2 different surface ablation methods. Saudi Med J 2015;36(1):67-72. doi:10.15537/smj.2015.1.9983.

25. Meek KM, Hayes S. Corneal cross-linking - a review. Ophthalmic Physiol Opt 2013;33(2):78-93. doi:10.1111/opo.12032.

26. Wollensak G, Herbst H. Significance of the lacunar hydration pattern after corneal cross linking. Cornea 2010;29:899-903. doi:10.1097/ICO.0b013e3181ca3293.

27. Koller T, Mrochen M, Seiler T. Complication and failure rates after corneal crosslinking. J Cataract Refract Surg 2009;35(8):1358-62. doi:10.1016/j.jcrs.2009.03.035.

28. Jester J V, Moller-Pedersen T, Huang J, et al. The cellular basis of corneal transparency: Evidence for "corneal crystallins". J Cell Sci 1999;112 (Pt 5):613-22.

29. Bovone C, Sparano MC, Balestrazzi A, et al. Corneal healing after riboflavin ultraviolet-A collagen cross-linking determined by confocal laser scanning microscopy in vivo: Early and late modifications. Am J Ophthalmol 2008;146:527-34. doi:10.1016/j. ajo.2008.05.042.

30. Greenstein SA, Fry KL, Bhatt J, et al. Natural history of corneal haze after collagen crosslinking for keratoconus and corneal ectasia: Scheimpflug and biomicroscopic analysis. J Cataract Refract Surg 2010;36(12):2105-14. doi:10.1016/j.jcrs.2010.06.067.

31. Connon CJ, Marshall J, Patmore AL, et al. Persistent haze and disorganization of anterior stromal collagen appear unrelated following phototherapeutic keratectomy. J Refract Surg 2002;19(3):323-32.

32. Teus MA, de Benito-Llopis L, Alió JL. Mitomycin C in corneal refractive surgery. Surv Ophthalmol 2009;54(4):487-502. doi:10.1016/j.survophthal.2009.04.002.

33. Kanellopoulos AJ, Binder PS. Collagen cross-linking (CCL) with sequential topography-guided PRK: A temporizing alternative for keratoconus to penetrating keratoplasty. Cornea 2007;26(7):891-5. doi:10.1097/ICO.0b013e318074e424.

34. Güell JL, Verdaguer P, Elies D, et al. Late onset of a persistent, deep stromal scarring after PRK and corneal cross-linking in a patient with forme fruste keratoconus. J Refract Surg 2014;30(4):286-8. doi:10.3928/1081597X-20140320-09.

35. Kymionis GD, Portaliou DM, Diakonis VF, et al. Posterior linear stromal haze formation after simultaneous photorefractive keratectomy followed by corneal collagen cross-linking. Invest Ophthalmol Vis Sci 2010;51(10):5030-3. doi:10.1167/iovs.09-5105

36. Jabbur NS, Sakatani K, O'Brien TP. Survey of complications and recommendations for management in dissatisfied patients seeking a consultation after refractive surgery. J Cataract Refract Surg 2004;30(9):1867-74. doi:10.1016/j.jcrs.2004.01.020.

37. Quinto GG, Camacho W, Behrens A. Postrefractive surgery dry eye. Curr Opin Ophthalmol 2008;19(4):335-41.

38. Ang RT, Dartt DA, Tsubota K. Dry eye after refractive surgery. Curr Opin Ophthalmol 2001;12:318-22. doi:10.1097/00055735200108000-00013.

39. Dartt DA. Dysfunctional neural regulation of lacrimal gland secretion and its role in the pathogenesis of dry eye syndromes. Ocul Surf 2004;2(2):76-91. doi:10.1016/S1542-0124(12)70146-5.

40. Kymionis GD, Mikropoulos DG, Portaliou DM, et al. An overview of corneal collagen cross-linking (CXL). Adv Ther 2013;30(10):858-69. doi:10.1007/s12325-013-0065-9.

41. Murakami Y, Manche EE. Prospective, randomized comparison of self-reported postoperative dry eye and visual fluctuation in LASIK and photorefractive keratectomy. Ophthalmology 2012;119(11):2220-4. doi:10.1016/j.ophtha.2012.06.013.

42. Kontadakis GA, Kymionis GD, Kankariya VP, et al. Effect of corneal collagen cross-linking on corneal innervation, corneal 
sensitivity, and tear function of patients with keratoconus. Ophthalmology 2013;120:917-22. doi:10.1016/j.ophtha.2012.10.012.

43. Dash SK. Corneal sensitivity is reduced and relates to the severity of neuropathy in patients with diabetes: response to Tavakoli et al. Diabetes Care 2007;30:e142; author reply e143. doi:10.2337/ dc07-1786.

44. Cruzat A, Pavon-Langston D, Hamrah P, et al. In vivo confocal microscopy of corneal nerves: Analysis and clinical correlation. Semin Ophthalmol 2010;25(5-6):171-7. doi:10.3109/08820538.201 0.518133 .

45. Jaanus SD. Ocular side effects of selected systemic drugs. Optom Clin 1992;2(4):73-96. http://ovidsp.ovid.com/ovidweb.cgi?T=JS\& $\mathrm{PAGE}=$ reference $\& D=$ emed $2 \& \mathrm{NEWS}=\mathrm{N} \& A \mathrm{~N}=1363080$.

46. Wong J, Lan W, Ong L. Non-hormonal systemic medications and dry eye. Ocul Surf 2010;9(4):1408-11. doi:10.1016/S15420124(11)70034-9.

47. Stapleton F, Marfurt C, Golebiowski B, et al. The TFOS International Workshop on Contact Lens Discomfort : Report of the subcommittee on neurobiology. Invest Ophthalmol Vis Sci 2013;54(11):TFOS71-97. doi:10.1167/iovs.13-13226.

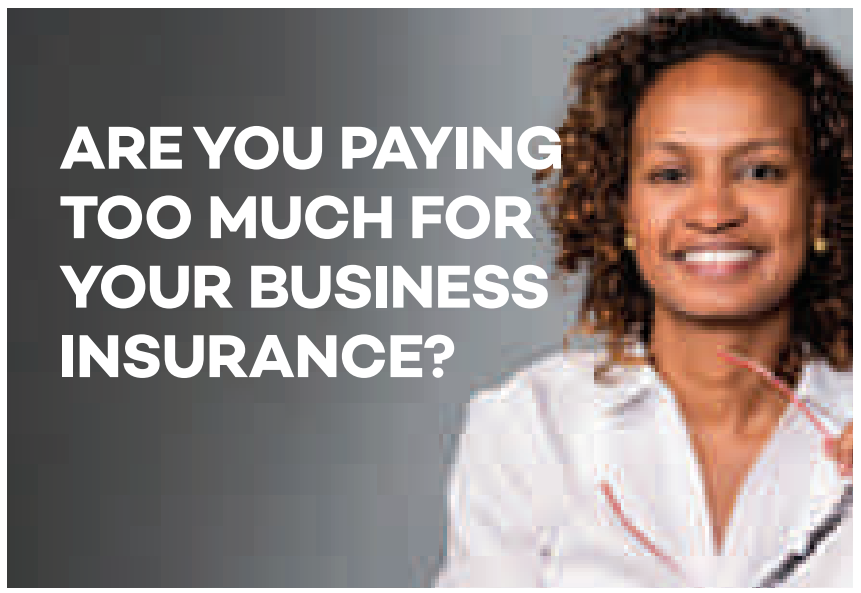

Find out how CAO members have increased coverage while saving an average of $40 \%$ on their insurance costs.

Visit www.cao.bmsgroup.com or contact a BMS broker at 1-844-517-1371 or cao.insurance@bmsgroup.com to secure a quote today!

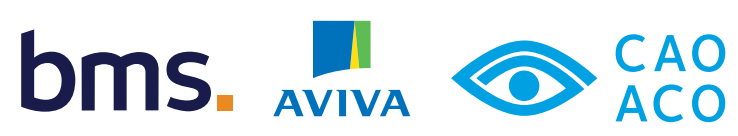

Your partners in protection 